\title{
A 32-YEAR-OLD OBESE MALE WITH PROLONGED INCREASED TRANSAMINASE LEVEL ASSOCIATED WITH NASH AFTER HBV HEPATITIS
}

\author{
AHMEDUL KABIR ${ }^{1}$, JAYANTA BANIK ${ }^{2}$, ROBED AMIN ${ }^{1}$, FAIZUL ISLAM CHOWDHURY ${ }^{3}$
}

\begin{abstract}
Nonalcoholic steatohepatitis (NASH) is an underdiagnosed liver disease and is the main cause of persistent elevated serum liver enzymes among the general population. Here, we report the case of a 32-year-old obese man who was diagnosed as a case of chronic hepatitis caused by HBV infection. He was treated with Lamivudine for two years. Though his HBV DNA load became negative, he had persistently elevated ALT and AST for two years. Then a liver biopsy was planned for greater diagnostic and prognostic certainty, as the patient was obese, and aspartate transaminase to alanine transaminase ratio was greater than one. The histopathology showed steatosis, ballooning degeneration, microinflammation and fibrosis compatible with non-alcoholic steatohepatitis. We present the case as we think that clinicians should be aware of the possibility, that hepatitis B viral hepatitis with prolonged elevated serum liver enzymes might be associated with non-alcoholic steatohepatitis.
\end{abstract}

Keyword: Non-alcoholic fatty liver, steatohepatitis

\section{Introduction}

Nonalcoholic steatohepatitis (NASH) is an underdiagnosed liver disease which is characterized by the histological features of steatohepatitis in the absence of significant alcohol consumption. ${ }^{1,2}$ Generally it is mild and slowly progressive but may eventually develop into cirrhosis and hepatocellular carcinoma. ${ }^{1,3}$ It is highly prevalent among individuals who are obese and have type 2 diabetes mellitus with metabolic syndrome. Insulin resistance, is the main mechanism of underlying metabolic syndrome and NASH, which is significantly higher in patients with HCV-related chronic hepatitis than in patients with HBV-related chronic liver disease. ${ }^{4}$ Nonalcoholic fatty liver (NAFL) is a precursor of NASH and is the main cause of elevated serum liver enzymes among the general population. ${ }^{1}$ Ludwig first described the pathology of NASH, characterised by macrovesicular steatosis, hepato- cellular ballooning and mild lobular inflammation with scattered polymorphonuclear leucocytes and monocytes, perisinusoidal or pericellular (chickenwire) fibrosis particular in acinar zone with occasional Mallory Bodies. ${ }^{1}$ A liver biopsy is required for its diagnosis as ultrasonography often misses the hepatic steatosis. Serum aminotransferases are elevated in most patients with NASH and they may have some degree of hepatomegaly but normal liver enzymes and a normal liver size do not exclude the diagnosis. Persistently elevated liver enzymes in target groups, such as obese persons, patients with type 2 diabetes mellitus, the level of suspicion should be raised for the frequent diagnosis of NASH. ${ }^{1}$

\section{Case Report}

A 32 years old obese man presented to our institution with persistently elevated ALT and AST for two years. He was diagnosed as a case of chronic hepatitis caused by HBV infection and was treated with Lamivudine for two years. Though his HBV DNA load became negative, serum ALT and AST did not normalize. Review of systems raveled no significant symptoms and there was no significant past medical history and family history regarding the illness.

On examination his BMI was $29.8 \mathrm{Kg} / \mathrm{m}^{2}$ and there is no other abnormality. On investigation, Complete

1. Assistant Professor, Department of Medicine, DMCH

2. Post-graduate Trainee, Dept. of Medicine, BSMMU

3. Associate Professor, Department of Medicine, DMCH

Correspondence : Dr. Ahmedul Kabir, Assistant Professor, Department of Medicine, DMCH 
blood count and Blood film revels, WBC $-12 \times 10^{9} /$ $\mathrm{L}$ with a PMN predominance of $72 \%$, Hematocrit $43 \%$, Platelets $-287 \times 10^{9} / \mathrm{L}, \mathrm{ESR}-20 \mathrm{~mm} / 1 \mathrm{st} \mathrm{Hr}$, $\mathrm{Hb}$ (Cyn. Meth.) - $10.6 \mathrm{~g} / \mathrm{dl}$, PBF: RBC - Normocytic and normochromic, WBC-Mature with Neutrophil Leukocytosis , Plateletes - normal. Urinalysis is negative for ketone bodies, protein or red cells. LFT shows, S.Bilirubin $0.8 \mathrm{mg} / \mathrm{dl}$, ALT(SGPT) $-87 \mathrm{U} / \mathrm{L}$, AST - 102 U/L, Prothrombin time - 12.0 sec ( control $12 \mathrm{sec})$, S. Total protein $-8.8 \mathrm{gm} / \mathrm{dl}$, S. Albumin $4.8 \mathrm{gm} / \mathrm{dl}$, S. Globulin $-3.0 \mathrm{gm} / \mathrm{dl}$ and S. AG ratio $1.6: 1$. HBsAg was positive amd anti $\mathrm{HCV} \mathrm{Ab}$ is negative. HBeAg was negative and HBV DNA load was $1.4 \times 10^{3}$ copies $/ \mathrm{ml}$. Fasting blood glucose is 4.44 $\mathrm{mmol} / \mathrm{L}$ and 2 hours after breakfast is $6.23 \mathrm{mmol} / \mathrm{L}$. Fasting lipid profile shows S. Total Cholesterol -4.81 $\mathrm{mmol} / \mathrm{L}, \mathrm{S}$. HDL $-0.98 \mathrm{mmol} / \mathrm{L}, \mathrm{S} . \mathrm{LDL}-2.95 \mathrm{mmol} /$ $\mathrm{L}$ and $\mathrm{S}$. TG $-2.62 \mathrm{mmol} / \mathrm{L}$. USG of whole abdomen shows normal liver size with homogenous paranchymal echo. Chest radiograph was normal. ECG and echocardiogram is within normal limit. Endoscopy of upper GIT is normal. Liver biopsy shows preservation of lobular architecture. Moderate steatosis consisting of both macro and micro-vesicular type, predominantly macrovesicular is present. Occasional hepatocytes show ballooning degeneration. Mild degree of lobular inflammation is present. The portal tract contains a small number of chronic inflammatory cells. No evidence of piecemeal necrosis or bridging fibrosis is noted.

\section{Discussion}

Nonalcoholic steatohepatitis (NASH) is a severe form of nonalcoholic fatty liver disease (NAFLD).$^{3}$ NAFL is a benign and reversible condition characterised by steatosis without inflammation or fibrosis although necroinflammation and some degree of pericellular fibrosis is seen in $20 \%$ of cases in liver histology. This is called nonalcoholic steatohepatitis (NASH) the precursor of which is NAFL. 20\% of NASH progresses to cirrhosis. To complete the terminology, both NAFL and NASH are called nonalcoholic fatty liver diseases (NAFLD). ${ }^{1}$

The aetiological risk factors for NASH are many. These include presence of family history of dyslipidaemia and cryptogenic cirrhosis, morbid obesity and hepatotoxic alcohol consumption. ${ }^{5}$ There is association between pituitary dysfunction and NASH has been described in patients with hypopituitarism. ${ }^{6}$ It is closely linked to nutrition possibly high-fructose corn syrup consumption and consumption of certain types of fats. In certain underdeveloped countries, industrial toxins may play a role in NASH by proinflammatory cytokines and oxidative stress, and there is increasing interest in the potential interaction of industrial toxins and nutrients. ${ }^{7}$ Some drugs may also cause NASH which includes oral hypoglycemic agents, lipid lowering agents, antihypertensives, and antiobesity medication with well-recognized hepatotoxicity. ${ }^{8}$ NASH may also develop in myeloproliferative disorder like chronic neutrophilic leukemia by infiltration of abnormal leukemic cells in the liver. ${ }^{9}$ Different cases illustrate the concepts of overlapping aetiologies of NASH like hepatitis $\mathrm{C}$, diabetes and lipodystrophy. The relationships between cryptogenic cirrhosis, familial cirrhosis, non-alcoholic fatty liver disease and hepatocellular carcinoma; familial hypobetalipoproteinaemia as an aetiology of steatohepatitis have also been described. ${ }^{5}$ Some reports showed that, sex and age, diabetes and BMI were independent risk factors for more severe liver fibrosis in HCV-positive patients, whereas they were not associated with the severity of liver fibrosis in HBV subjects. ${ }^{4}$

In the pathogenesis of NASH two steps or 'hits' is recognized. Fat accumulation in the liver is the 'first hit'. Free fatty acids are taken up in the hepatocytes where they are either metabolised via peroxisomal or mitochondrial-oxidation or stored as triglycerides. VLDL is the rate-determining step in triglyceride export from the liver. In NASH its synthesis is decreased. The insulin-resistant state favours lipolysis in the adipose tissues, FFA synthesis and lipogenesis in the liver. The net result is hepatic fat accumulation. Reactive oxygen species (ROS) and inflammation represent the 'second hit'. Continuous and superfluous FFA oxidation causes the generation of ROS in excess with oxidant stress as a result. Cytokines are produced in hepatocytes as well as in Kupffer cells. TGF stimulates hepatic stellate cells to produce collagen and cause liver fibrosis, in addition these cells transform into myofibroblasts with more collagen formation and an increase of intrahepatic vascular resistance and portal hypertension. Chemokines attract monocytes and neutrophils with inflammation, more oxidant stress, hepatocellular apoptosis and injury as a result. ${ }^{1}$

Conditions with bacterial overgrowth such as jejunoileal bypass cause NASH which suggests that 
intestinal dysmotility and bacterial overgrowth of the small intestine may also contribute to NASH. It can be hypothesised that lipohilic endotoxins from the gut may accumulate in the fat of hepatocytes from where they are slowly but continuously released which stimulates Kupffer cells to produce TNF, interleukin 1 and 6 and the profibrotic cytokine TGF and TGF transforms the hepatic stellate cells into collagen-producing myofibroblasts. In addition, monocytes and neutrophils are attracted by chemokines and increases the oxidative stress and cell injury. ${ }^{1}$

Adiponectin (a hormone produced by peripheral adipose tissue) deficiency may be important in the development of NASH. Adiponectin increases fatty acid oxidation thereby decreasing the hepatic triglyceride content and ameliorating insulin resistance. Other studies also show that adiponectin decreases necrosis and inflammation in the liver. In $\mathrm{NASH}$, adiponectin production is decreased and the liver is vulnerable to the action of cytokines and ROS in the absence of this protective factor. ${ }^{1}$

NASH is commonly accompanied with diabetes mellitus (especially type II), obesity and hyperlipidemia. ${ }^{10}$ Liver biopsy is required for the diagnosis of NASH as it is an underdiagnosed disease. Most patients with NASH have elevated serum aminotransferases and diagnostic imaging like ultrasonography often misses the steatohepatitis . ${ }^{1}$ It may be accompanied with other causes of hepatitis like HBV and HCV chronic hepatitis. FibroScan replaces the liver biopsy in the evaluation of patients with chronic hepatitis B or C, but important additional liver disease like non-alcoholic steatohepatitis or hepatic steatosis might be missed, in the majority of these cases. So liver biopsy should be considered in patients with chronic hepatitis B or $\mathrm{C}$ having elevated liver enzyme and who are obese with type 2 diabetes mellitus and who are on total parenteral nutrition. ${ }^{1,11}$ Magnetic resonance proton spectroscopy of the liver may be a noninvasive procedure to detect and quantify liver fat. ${ }^{1}$ Highsensitivity $\mathrm{CRP}(\mathrm{hs}-\mathrm{CRP})$ concentration may be a promising biomarker for screening of NASH as it is positive in higher concentration in patients with more active form of NASH than those with quiescent form of NASH. ${ }^{12}$

Features found in biopsy specimen are steatosis, polymorphonuclear lobular inflammation, ballooning degeneration, hyaline- Mallory bodies and cirrhosis. Although NASH may coexist with other chronic liver diseases including drug induced liver disease, chronic viral hepatitis, and metabolic and autoimmune liver diseases, it is important to rule out them to establish the diagnosis of NASH. ${ }^{10}$ Liver histopathology is very similar in alcoholic liver disease and NASH. Cholestasis, central-central and central-portal bridging necrosis frequently occurs in alcoholic liver disease but is uncommon in NASH. All features of NASH may be present in hepatitis C. Ballooning degeneration, Mallory's hyaline and fibrosis are discriminating features of progressive NASH, but in hepatitis $\mathrm{C}$ the inflammation and fibrosis are mainly localized in the portal or periportal areas. ${ }^{1}$

There is no definite treatment for NASH and therapeutic measures are taken to reduce the risk factors of NASH by using hepatocellular protective agents. ${ }^{10}$ Because NASH has the potential to progress to cirrhosis, treatment of NASH is considered in patients with more advanced disease on biopsy. ${ }^{13}$ The measures include weight reduction, treating hyperinsulinemia and diabetes, control of hypertriglyceridemia and leptin. ${ }^{10}$ Lifestyle adjustments should be tried first: which include weight reducing diets with polyunsaturated fatty acids and programmed exercise. Excercise diverts the fatty acids away from the liver to be metabolised in the muscles and it decreases insulin resistance. Polyunsaturated fatty acids stimulate PPARa and repress the sterol regulatory element-binding protein SREBP-1 which reduced both hepatic steatosis and insulin resistance. ${ }^{1}$ Gradual weight loss and exercise have significant decreases in ALT and AST levels with improvement of steatosis and fibrosis in NASH patients but rapid weight loss particularly with bariatric surgery, may acutely worsen NASH. Weight loss should be approximately 1 to $2 \mathrm{lb}$ (0.45 to 0.90 $\mathrm{kg}$ ) per week. ${ }^{13}$

Drug therapy includes anti-inflammatory, antioxidative, cytoprotective and lipolytic agents but none have been shown to be very effective. They should aim at several levels and it is unlikely that a single drug could do the job, for example supplementation with vitamin $\mathrm{E}$ is ineffective but vitamin $\mathrm{E}$ in combination with vitamin $\mathrm{C}$ has shown to reduce fibrosis. ${ }^{1}$ Treatment of insulin resistance has improved disease-oriented outcomes in patients with NASH and current trials focus on drugs that 
increase insulin sensitivity. ${ }^{1,13}$ Patients with NASH who did not have diabetes and received metformin had significant reductions in AST and ALT levels and decreased hepatic steatosis. ${ }^{13}$ The thiazolidinediones are PPARg ligand and have a direct effect on PPARg in hepatic stellate cells which retards collagen synthesis and fibrosis both in liver. ${ }^{1}$ Rosiglitazone improves steatosis and fibrosis, reduce AST, ALT levels and necroinflammatory score, but associated with an undesirable weight gain. ${ }^{1,13}$ Pioglitazone caused significant decreases in ALT, AST, insulin, and C-peptide levels; steatosis decreased and liver histology improved. ${ }^{13}$ Medications for treating hyperlipidemia like atorvastatin and pravastatin on patients with NASH showed improvement in liver histology and liver enzymes, but there is no evidence that improving biochemical measures reduces morbidity or mortality rates. ${ }^{13}$ If a drug is suspected as a cause of NASH, it is probably prudent to stop this medication. ${ }^{8}$ Drugs that stimulate adiponectin release from adipose tissue and drugs that stimulate b-oxidation in muscle, may be used for treatment of NASH and is a promising field for future research. ${ }^{1}$ Finally, decompensated NASH-related cirrhosis is an indication for liver transplantation. ${ }^{1}$

\section{Conclusion}

Non-alcoholic steatohepatitis (NASH) is a disease of unknown origin characterized histologically by fatty infiltration with ballooning degeneration and with lesions similar to alcoholic hepatitis and sinusoidal fibrosis, polymorphonuclear infiltration with or without Mallory hyaline. NASH is frequently associated with disorders such as insulin resistance, obesity, type 2 diabetes mellitus, hyperlipidemia, protein-calorie malnutrition, and jejeunoileal bypass surgery. In the evaluation of patients with chronic hepatitis $\mathrm{B}$ or $\mathrm{C}$ not responding to standard antiviral therapy, important additional liver disease might be thought before thinking resistance to antiviral drugs. In the majority of these cases non-alcoholic steatohepatitis or hepatic steatosis is being missed. Therefore a liver biopsy should still be considered in a patient presenting with chronic hepatitis $\mathrm{B}$ or $\mathrm{C}$ with persistently elevated liver enzymes despite treatment, as NASH can progress to cirrhosis and hepatic failure. Furthermore understanding the importance of a potentially serious and lifethreatening disease, clinicians should be aware of the possibility that prolonged liver enzyme abnormalities in chronic hepatitis might be associated with non-alcoholic steatohepatitis.

\section{References}

1. Jansen PLM. Nonalcoholic steatohepatitis. The Netherlands Journal of Medicine 2004;62:7/8

2. Yoshioka Y, Hashimoto E, Yatsuji S, et al. Nonalcoholic steatohepatitis: cirrhosis, hepatocellular carcinoma, and burnt-out NASH. J Gastroenterol 2004; 39: 1215-1218.

3. Tseng PH, Liu CJ, Kao JH, et al. Disease Progression in a Patient with Nonalcoholic Steatohepatitis. Journal of the Formosan Medical Association 2008; 107(10): 816-821.

4. Persico M, Masarone M, Mura VL, et al. Clinical expression of insulin resistance in hepatitis $\mathrm{C}$ and $B$ virus-related chronic hepatitis: Differences and similarities. World J Gastroenterol 2009; 15(4): $462-466$.

5. Lonardo A, Bagni A, Tarugi P, et al. The wide spectrum of steatohepatitis: a report of four cases and a review of the literature. Eur J Gastroenterol Hepatol 2004; 16(10): 1043-1050.

6. Nakajima K, Hashimoto E, Kaneda H, et al. Pediatric nonalcoholic steatohepatitis associated with hypopituitarism. J Gastroenterol 2005; 40(3): 312-315.

7. Cave M, Deaciuc I, Mendez C, et al. Nonalcoholic fatty liver disease: predisposing factors and the role of nutrition. J Nutr Biochem. 2007; 18(3):184-95.

8. SK Das, DM Vasudevan. Drugs and non-alcoholic steatohepatitis. Indian J Pharmacol 2006; 38: 238-42.

9. Yoshida C, Kojima H, Iijima T, et al. Association of non-alcoholic steatohepatitis (NASH) with chronic neutrophilic leukemia. Eur J Haematol 2004; 72(3): $225-228$.

10. Review Article Non-Alcoholic Steatohepatitis (NASH); Govaresh 2004; 9: 110-21.

11. Verveer C, Zondervan PE, Janssen HL. The presence of additional diagnoses in patients with chronic hepatitis B and C. Histological evaluation of liver biopsies used for correlation with Transient Elastography in 257 patients; Diagnostic Tools Biochemical/Imaging; 1946; Co-morbidities and Non-B/Non-C Hepatitis Viruses.

12. Uchihara M, Izumi N. High-sensitivity C-reactive protein (hs-CRP): a promising biomarker for the screening of non-alcoholic steatohepatitis (NASH). Nippon Rinsho 2006; 64(6): 1133-8.

13. Bayard M, Holt J, Boroughs E. Nonalcoholic Fatty Liver Disease. Am Fam Physician 2006; 73(11). 